\title{
Predictors of death in patients with COVID-19: A cross-sectional study in West of Iran
}

\author{
Manoochehr Karami ${ }^{1,2}$, Mohammad Mirzaei ${ }^{3}$, Fatemeh Shahbazi ${ }^{1,4}$, Fariba Keramat ${ }^{5}$, Ebrahim Jalili ${ }^{6}$, Saeid Bashirian $^{7}$, \\ Rashid Heidarimoghadam ${ }^{8}$, Jalal Bathaei ${ }^{3}$, Salman Khazaei ${ }^{9,10 *(1)}$
}

Received: 23 Jul 2020

Published: 11 Aug 2021

\section{Abstract}

Background: Coronavirus disease 2019 (COVID-19) is a contagious disease caused by a newly identified coronavirus. Our knowledge about the survival rate and prognostic factors of the disease is not established well. The purpose of this study was to evaluate the predictors of COVID-19 mortality in Hamadan province in western Iran.

Methods: In this study, we included all laboratory-confirmed COVID-19 cases with known treatment outcomes in Hamadan province, Iran, between 20, 2020, to May 10, 2020. Demographic, clinical, laboratory data, and treatment outcomes were obtained from computerized medical records and compared between survived cases and patients with death outcomes. Univariable and multivariable logistic regression models were used to determine the predictors of death.

Results: From 749 investigated patients, 77 patients (10.28\%) died during the treatment. The Mean age of patients was $53.97 \pm 19.04$ years. Multivariable logistic regression showed that males had 2.07 (95\% CI: 1.73, 2.54) fold higher odds of death. Those with 60 years old and more had 6.49 (95\% CI: 4.53, 7.93) fold higher odds of death. Patients with an underlying disease had 7.14 (95\% CI: $6.94,7.38)$ fold higher odds of death, and patients who were hospitalized in the ICU ward had 2.24 (95\% CI: 1.75, 2.90) times higher odds of COVID-19 related mortality.

Conclusion: The potential predictors of death in COVID-19 cases, including the male gender, older age, and having an underlying disease could help physicians to identify patients with poor prognoses at an early stage and better management of them.

Keywords: Coronavirus, COVID-19, Epidemiology, Mortality, Iran

Conflicts of Interest: None declared

Funding: The study was funded by the Hamadan University of Medical Sciences, Hamadan, Iran (No. 140002211138). The funder hiad no role in the design of the study, data collection, analysis, interpretation of data, and writing the manuscript.

\section{*This work has been published under CC BY-NC-SA 1.0 license.}

Copyright $\odot$ Iran University of Medical Sciences

Cite this article as: Karami M, Mirzaei M, Shahbazi F, Keramat F, Jalili E, Bashirian S, Heidarimoghadam R, Bathaei J, Khazaei S. Predictors of death in patients with COVID-19: A cross-sectional study in West of Iran. Med J Islam Repub Iran. 2021 (11 Aug);35:103. https://doi.org/10.47176/mjiri.35.103

\section{Introduction}

An outbreak of coronavirus disease 2019 (COVID-19)

Corresponding author: Dr Salman Khazaei, s.khazaei@umsha.ac.ir

1. Department of Epidemiology, School of Public Health, Hamadan University of Medical Sciences, Hamadan, Iran

2. Modeling of Noncommunicable Disease Research Center, School of Public Health, Hamadan University of Medical Sciences, Hamadan, Iran

3. Deputy Minister of Health, Hamadan University of Medical Sciences, Hamadan, Iran

4. Students Research Committee, Hamadan University of Medical Sciences, Hamadan, Iran

5rucellosis Research Center, Hamadan University of Medical Sciences, Hamadan, Iran

6. Department of Emergency Medicine, School of Medicine, Hamadan University of Medical Sciences, Hamadan, Iran

7. Social Determinants of Health Research Center, Hamadan University of Medical Sciences, Hamadan, Iran

8. Department of Ergonomics, School of Health, Hamadan University of Medical Sciences, Hamadan, Iran

9. Research Center for Health Sciences, Hamadan University of Medical Sciences, Hamadan, Iran

10. Autism Spectrum Disorders Research Center, Hamadan University of Medical Sciences, Hamadan, Iran infection first emerged in late 2019 in Wuhan, China re-

$\uparrow$ What is "already known" in this topic:

A critical point for better management of COVID-19 is identifying the factors affecting patient mortality by each geographical area.

$\rightarrow$ What this article adds:

Analyzing the data from 749 confirmed cases indicated that men, the elderly over 60 years old, people with underlying diseases and patients who were admitted to the ICU unit were more likely to die from coronavirus infection. 
sulting in more than 80000 confirmed cases in this country and being exported to a growing number of countries (1). World Health Organization (WHO) declares a global public health emergency over the COVID-19 outbreak on 30 January 2020. Alongside severe acute respiratory syndrome (SARS) and the Middle East respiratory syndrome (MERS), COVID-19 is another common type of coronavirus that infects humans (2).

Although the outbreak is possible to have originated from a zoonotic transmission event correlated with a great seafood marketplace that too traded in live wild animals, it quickly converted clear that efficient person-to-person transmission was also happening (3). The clinical spectrum of SARS-CoV-2 infection seems to be widespread, including asymptomatic infection, mild upper respiratory tract disorder, and severe viral pneumonia with respiratory malfunction and also death (4-6). Based on previous epidemiological research, mortality is higher between men, the elderly, and residents in rural area. The typical clinical characteristics of subjects with COVID-19 were weakness, fever, dry cough, and dyspnea (5-7).

It was published that existing diseases such as hypertension and diabetes mellitus are important risk factors for the fatality of patients affected by $\operatorname{COVID}-19(5,6,8)$. To the best of the author's knowledge, no earlier studies have been committed to established death cases in Hamadan province. Also, the epidemiological and clinical features of approved death cases in the Hamadan province have not yet been described. Therefore, the epidemiology and clinical characteristics of death cases with COVID-19 were summarized with the intent of early identification of critically ill patients, thereby reducing mortality. To understand the characteristics of patients who die of COVID-19, we evaluated the outcome of 749 COVID-19 confirmed cases in the west of Iran.

\section{Methods}

This study was conducted on 749 confirmed COVID-19 cases according to WHO interim guidance, treated in all hospitals affiliated to Hamadan University of Medical Sciences between February 20, 2020, and May 10, 2020. In this study, a known treatment outcome was considered as inclusion criteria, and those who died or were discharged with good condition were entered into the study.

The protocol of the study was approved by the Ethics Committee of Hamadan University of Medical Sciences. In this study, the diagnosis of COVID-19 was based on the result of real-time reverse-transcriptase polymerasechain-reaction (RT-PCR) detection and cases with positive RT-PCR on samples collected from upper respiratory oropharyngeal swabs, with or without nasopharyngeal swabs and sputum were considered as a confirmed case.

A standardized data collection tool contains demographic characteristics, clinical presentation, laboratory findings, chest CT examination, and disease outcomes of identified cases were used for collecting data. Demographic and clinical data including signs, symptoms and underlying disease history, were asked directly from the patients and when the patient was unable to respond due to a bad physical condition, the information was taken with the physician, his/her families or referring to the patients medical records. Biochemical blood test results were gathered by referring to the patient's medical record. Finally, the gathered data was transported to the deputy of health.

Descriptive statistics were reported as number (\%) for categorical variables and mean (SD) for continuous variables across the patient's background. Univariable logistic regression was conducted to estimate the crude association between demographic, laboratory, and clinical characteristics of patients and disease outcomes (Remission/ Death). Those with $\mathrm{P}$-value $<0.2$ were considered as potential significant determinants of death and were included in multivariable logistic regression. All statistical analyses were conducted in Stata version 14 software. A significant level was considered less than $5 \%$.

\section{Results}

Up to April 20, 2020, a total of 749 confirmed COVID19 patients were diagnosed in hospitals, as well as outpatient care centers affiliated to Hamadan University of Medical Sciences. Of them, 77 patients died during the treatment (case fatality rate: $10.28 \%$ ).

Baseline characteristics of identified COVID-19 cases are shown in Figure 1. The number of 375 (50.07\%) cases were males, and $529(70.63 \%)$ were urban dwellers. The Mean age of them was $53.97 \pm 19.04$ years and in total, only $16(2.14 \%)$ of patients were under 20 years of age.

A full comparison of demographic and clinical variables of survived cases compared with those dead, as well as the results of univariable logistic regression analyses associated with death, are presented in Table 1. In univariable analysis male gender (OR: 1.75, $95 \mathrm{CI}: 1.08,2.95)$, age groups 60 years and older (OR: 12.26, $95 \mathrm{CI}: 11.12$, 13.42), having an underlying disease (OR: 9.58, 95\% CI: 8.84, 10.14), abnormal lung C'T scan findings (OR: 2.67, $95 \mathrm{CI}: 1.69,3.55)$, having chest pain (OR: 2.27, $95 \mathrm{CI}$ : $1.08,4.06)$ and hospitalized in the ICU ward (OR: 2.24, 95 CI: $1.75,2.90)$, were potentially associated with higher odds of death in COVID-19 cases $(p<0.05)$.

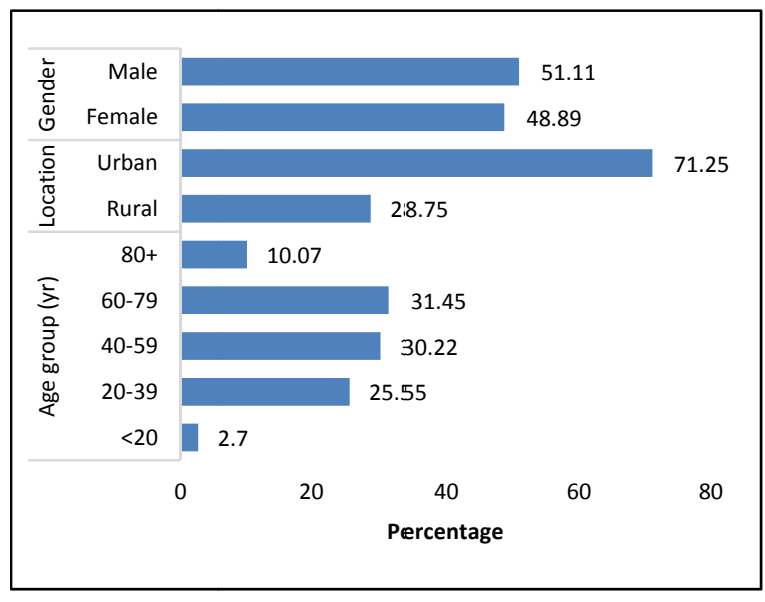

Fig. 1. Baseline characteristics of identified COVID-19 cases with known treatment outcomes in Hamadan province 
Table 1. Comparison of demographic and clinical characteristics between recovered and dead COVID-19 cases as well as univariable logistic regression

\begin{tabular}{|c|c|c|c|c|c|}
\hline \multirow[t]{2}{*}{ Variable } & \multirow{2}{*}{$\begin{array}{c}\text { Recovered } \\
\mathrm{N}=672(89.72 \%)\end{array}$} & \multirow{2}{*}{$\begin{array}{c}\text { Death } \\
\mathrm{N}=77(10.28 \%)\end{array}$} & \multirow[t]{2}{*}{$\mathrm{p}$} & \multicolumn{2}{|c|}{ Crude model } \\
\hline & & & & OR $(95 \% \mathrm{CI})$ & $\mathrm{p}$ \\
\hline \multicolumn{6}{|l|}{ Gender } \\
\hline Female & $345(92.25)$ & $29(7.75)$ & 0.023 & References & 0.024 \\
\hline Male & $327(87.20)$ & $48(12.80)$ & & $1.75(1.08,2.84)$ & \\
\hline \multicolumn{6}{|c|}{ Age group (Year) } \\
\hline$<40$ & $197(98.01)$ & $4(1.99)$ & 0.001 & References & \\
\hline $40-59$ & $230(95.04)$ & $12(4.96)$ & & $2.46(0.82,8.09)$ & 0.110 \\
\hline $60+$ & $245(80.07)$ & $61(19.93)$ & & $12.26(4.38,34.31)$ & 0.001 \\
\hline \multicolumn{6}{|l|}{ Location } \\
\hline Urban & $478(90.36)$ & $51(9.64)$ & 0.370 & References & 0.370 \\
\hline Rural & $194(88.18)$ & $26(11.82)$ & & $1.26(0.76,2.07)$ & \\
\hline \multicolumn{6}{|c|}{ Underling diseases } \\
\hline No & $501(96.53)$ & $18(3.47)$ & 0.001 & References & 0.001 \\
\hline Yes & $171(74.35)$ & $59(25.65)$ & & $9.58(5.50,16.70)$ & \\
\hline \multicolumn{6}{|c|}{ Lung CT Scan findings } \\
\hline Normal & $612(90.67)$ & $63(9.33)$ & 0.010 & References & 0.012 \\
\hline Abnormal & $60(81.08)$ & $14(18.92)$ & & $2.67(1.20,4.29)$ & \\
\hline \multicolumn{6}{|l|}{ Chest pain } \\
\hline No & $601(90.24)$ & $65(9.76)$ & 0.180 & References & 0.012 \\
\hline Yes & $71(85.54)$ & $12(14.46)$ & & $2.27(1.2,4.29)$ & \\
\hline \multicolumn{6}{|c|}{ Respiratory distress } \\
\hline No & $180(89.55)$ & $21(10.45)$ & 0.930 & References & 0.930 \\
\hline Yes & $492(89.78)$ & $56(10.22)$ & & $0.98(0.57,1.66)$ & \\
\hline \multicolumn{6}{|c|}{ Hospital ward } \\
\hline General & $531(91.71)$ & $48(8.29)$ & 0.001 & References & 0.001 \\
\hline ICU & $141(82.94)$ & $29(17.06)$ & & $2.27(1.38,3.73)$ & \\
\hline
\end{tabular}

Table 2 shows the results of the multivariate logistic regression. After adjusting for other variables, males had 2.09 fold higher odds of death ([OR=2.09, 95\% CI: 1.22 , $3.60)$ ], $\mathrm{p}=0.007)$. Compared to patients under 40 years of age, patients with 60 years and more had 6.49 fold higher odds of death $([\mathrm{OR}=6.49,95 \% \quad \mathrm{CI}: 2.23,18.88)]$, $\mathrm{P}=0.001$ ). Patients with an underlying disease had 7.14 fold higher odds of death $(\mathrm{p}<0.001)$, and patients who were hospitalized in ICU had 2.24 times higher odds of COVID-19 related mortality $(\mathrm{p}=0.005)$. As shown in Figure 2, the area under the curve (AUC) for assessing the discriminant power of the explanatory variables for COVID-19 related death is 0.85 , which indicates the entry of important variables into the model.

\section{Discussion}

This study proposed to determine risk factors associated with death in identified COVID-19 cases in Hamadan province. The demographic features such as age, sex, residence, cause of death, and comorbidities of the cases were obtained from the patients' medical records. In particular, the male gender, elderly over 60 years, having an underlying disease, and hospitalization in the ICU ward were associated with higher odds of COVID-19 related death.

The current study verified that increased age was associated with mortality in patients with COVID-19. This result is in concordance with the previous research, which demonstrated a higher case fatality rate between the elderly populations $(9,10)$. Elderly people, because they have a less capable cell-mediated immune response to infectious challenges, are more susceptible to infection. On the other hand, based on the previous research, aging has been expressed as an important independent risk factor for mortality in SARS-CoV and MERS- CoV. Early studies in ma- caques treated with SARS-CoV found that older macaques had more powerful host innate responses to virus infection

Table 2. Multivariable analysis of demographic and clinical characteristics associated with death from COVID-19

\begin{tabular}{lcc}
\hline & \multicolumn{2}{c}{ Multivariable model } \\
\cline { 2 - 3 } Variable & OR $(95 \% \mathrm{CI})$ & $\mathrm{p}$ \\
\hline Fender & References & 0.007 \\
Male & $2.09(1.22,3.60)$ & \\
Age group (Year) & & \\
$<40$ & References & 0430 \\
$40-59$ & $1.61(0.49,5.27)$ & 0.001 \\
$60+$ & $6.49(2.23,18.88)$ & \\
Underling diseases & References & 0.001 \\
No & $7.14(3.98,12.82)$ & \\
Yes & References & 0.005 \\
Hospital ward & $2.24(1.28,3.94)$ & \\
General & & \\
ICU & & \\
\hline
\end{tabular}

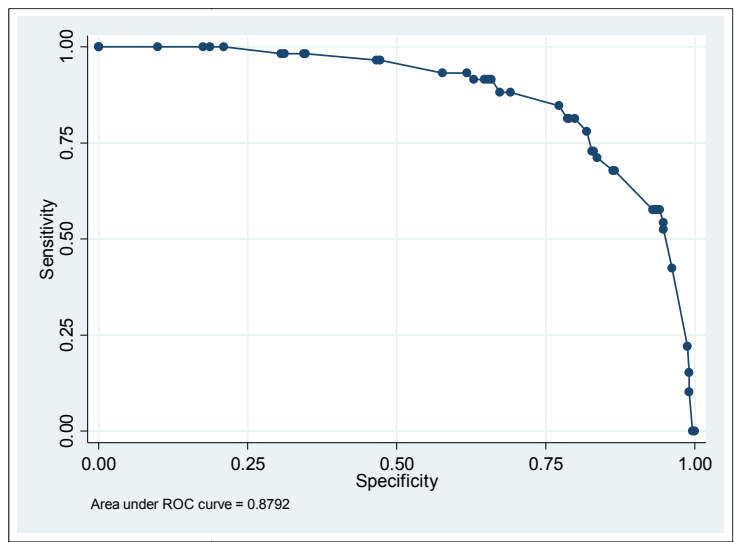

Fig. 2. The area under the curve (AUC) for assessing the discriminant power of the explanatory variables for COVID-19 related death 
than more juvenile adults, with an increase in differential expression of genes correlated with inflammation, whereas expression of type I interferon beta was decreased. The age-dependent defects in T-cell and B-cell function and the excess generation of type 2 cytokines could lead to a deficiency in control of viral replication and more prolonged proinflammatory responses, potentially leading to a poor outcome (11).

Analysis of data showed that the chance of death due to COVID-19 in men is 2.09 times higher than in women. Epidemiological studies show that males experiencing higher CFRs compared with females after COVID-19 infection $(12,13)$. Investigations from mainland China showed that men manifest more serious forms of the disease during the COVID-19 epidemic compared to women $(14,15)$. Male dominance in cases of COVID-19 has been reported by other countries as well (16-18). On the other hand, this limited sensitivity of women to viral contaminations can be assigned to the protection from sex hormones and the $\mathrm{X}$ chromosome, which play a crucial function in intrinsic and adaptive stability (5). From another perspective, a greater incidence rate of COVID-19 in males might be due to greater social communications in workplaces. Federal service for statistics in 2019 stated that men include 81 percent of the workforce in Iran, while more than 50 percent of them are engaged in service jobs. Consequently, there is a higher chance for men to get SARS$\mathrm{CoV}_{2}$ infection due to higher social interactions in work settings $(19,20)$.

According to the information presented in this article, more than $70 \%$ of the patients in this research were residents in urban areas. The accumulation of cases in urban areas may occur due to agents associated with access to healthcare or incomplete or poor surveillance and monitoring in rural areas $(21,22)$.

Another interesting finding of this research is that having an underlying disease increases the chance of death from COVID-19 (4-6). Based on the previous case series in 2020, the incidence of underlying diseases such as hypertension and subsequent hospitalization in the invasive care unit and death has been reported in patients with COVID-19 $(23,24)$.

In the present study, the proportion of death in patients with abnormal lung CT scans was significantly higher $(18.92 \%$ vs. $9.33 \%)$. According to the researches, severe acute respiratory syndrome coronavirus contaminates the lungs by the angiotensin-converting enzyme II receptor (25). More research is required to determine the mechanism of COVID-19. Also, clinical investigations are needed to verify whether angiotensin-converting enzyme inhibitors and angiotensin receptor blockers could stay profitable for cases with COVID-19.

The study has some limitations. Due to the retrospective study design, not all epidemiological, clinical, and laboratory data were done in all patients. Second, due to the small sample size, the external validity of the study decreases. However, by including all patients in the study population, we considered our study is representative of cases diagnosed and treated in Hamadan province. Third, our data were from patients who died during late April
2020, and they may not be representative of later cases of COVID-19. Fourth, there may occur selection bias because mainly patients with relatively severe COVID-19 pneumonia were hospitalized during this period and moderate patients are not admitted to the hospitals.

\section{Conclusion}

In conclusion, this study introduced several factors that associated with death from COVID-19 infection. The potential predictors of death in COVID-19 cases, including the male gender, older age, and having an underlying disease could help physicians to identify patients with poor prognoses at an early stage and better management of them.

\section{Acknowledgment}

Deputy of Research and Technology of Hamadan University of Medical Sciences approved our study (ID: IR.UMSHA.REC.1400.155). We would like to gratefully acknowledge all hospital staff involved in treating COVID-19 patients.

\section{Conflict of Interests}

The authors declare that they have no competing interests.

\section{References}

1. Shahbazi F, Solgi M, Khazaei S. Predisposing risk factors for COVID19 infection: a case-control study. Caspian J Intern Med. 2020;11(Suppl 1):495.

2. World Health Organization. Coronavirus disease 2019 (COVID-19): situation report-37.2020.

3. Thompson R. Pandemic potential of 2019-nCoV. Lancet Infect Dis. 2020;20(3):280.

4. Bai Y, Yao L, Wei T, Tian F, Jin DY, Chen L, et al. Presumed asymptomatic carrier transmission of COVID-19. JAMA. 2020;323(14):1406-7.

5. Chen N, Zhou M, Dong X, Qu J, Gong F, Han Y, et al. Epidemiological and clinical characteristics of 99 cases of 2019 novel coronavirus pneumonia in Wuhan, China: a descriptive study. Lancet. 2020;395(10223):507-13.

6. Huang C, Wang Y, Li X, Ren L, Zhao J, Hu Y, et al. Clinical features of patients infected with 2019 novel coronavirus in Wuhan, China. Lancet. 2020;395(10223):497-506.

7. Alipio M, Pregoner JD. Epidemiological characteristics of an outbreak of Coronavirus Disease 2019 in the Philippines. Available at SSRN 3568934. 2020.

8. Wang D, Hu B, Hu C, Zhu F, Liu X, Zhang J, et al. Clinical characteristics of 138 hospitalized patients with 2019 novel coronavirus-infected pneumonia in Wuhan, China. JAMA. 2020;323(11):1061-9.

9. Choi KW, Chau TN, Tsang O, Tso E, Chiu MC, Tong WL, et al. Outcomes and prognostic factors in 267 patients with severe acute respiratory syndrome in Hong Kong. Ann Intern Med. 2003;139(9):715-23.

10. Hong KH, Choi JP, Hong SH, Lee J, Kwon JS, Kim SM, et al. Predictors of mortality in Middle East respiratory syndrome (MERS). Thorax. 2018;73(3):286-9.

11. Zhou F, Yu T, Du R, Fan G, Liu Y, Liu Z, et al. Clinical course and risk factors for mortality of adult inpatients with COVID-19 in Wuhan, China: a retrospective cohort study. Lancet. 2020;395(10229): 1054-62.

12. Karlberg J, Chong DS, Lai WY. Do men have a higher case fatality rate of severe acute respiratory syndrome than women do? Am J Epidemiol. 2004;159:229-31.

13. Leong HN, Earnest A, Lim HH, Chin CF, Tan C, Puhaindran ME, et al. SARS in Singapore-predictors of disease severity. Ann Acad Med Singapore. 2006;35:326-31. 
14. Zhang DX, Cao YY, Yuan YD, Yang YB, Yan YQ, Akdis CA, et al. Clinical characteristics of 140 patients infected by SARS-CoV-2 in Wuhan, China. Allergy. 2020 Feb 19 doi: 101111/all14238 [Epub ahead of print].

15. Li Q, Guan X, Wu P, Wang X, Zhou L, Tong Y, et al. Early Transmission Dynamics in Wuhan, China, of Novel CoronavirusInfected Pneumonia. N Engl J Med. 2020;382:1199-207.

16. Chih-Cheng L, Tzu-Ping S, Wen-Chien K, Hung-Jen T, Po-Ren H. Severe acute respiratory syndrome coronavirus 2 (SARS-CoV-2) and coronavirus disease-2019 (COVID-19): The epidemic and the challenges. Int J Antimicrob Agents. 2020;55(105924).

17. Chen N, Zhou M, Dong X, Qu J, Gong F, Han Y, et al. Epidemiological and clinical characteristics of 99 cases of 2019 novel coronavirus pneumonia in Wuhan, China: a descriptive study. Lancet (London, England). 2020;395:07-13.

18. Zhang L, Yunhui L. Potential interventions for novel coronavirus in China: A systematic review. J Med Virol. 2020.

19. Hooper GL. Health Seeking in Men: A Concept Analysis. Urol Nurs. 2016;36(4)

20. Shahbazi F, Karami M, Mirzaei M, Mohammadi Y. Survival Rates and Prognostic Factors in Patients with Coronavirus Disease 2019: A Registry-Based Retrospective Cohort Study. J Res Health Sci. 2021.

21. Xu RH, He JF, Evans MR, Peng GW, Field HE, Yu DW, et al. Epidemiologic clues to SARS origin in China. Emerg Infect Dis. 2004;10(6):1030.

22. Shahbazi F, Khazaei S. Socio-economic inequality in global incidence and mortality rates from coronavirus disease 2019: an ecological study. New Microbes New Infect. 2020;38:100762.

23. Xie J, Wang H, Kang Y, Zhou L, Liu Z, Qin B, et al. The epidemiology of sepsis in Chinese ICUs: a national cross-sectional survey. Crit Care Med. 2020;48(3):e209-e18.

24. Shahbazi M, Shahbazi F. COVID-19 and children with special healthcare needs: Recommendations for helping parents and caregivers of young children. Arch Pediatr Infect Dis. 2021;9(1):1-3.

25. Zhao Y, Zhao Z, Wang Y, Zhou Y, Ma Y, Zuo W. Single-cell RNA expression profiling of ACE2, the receptor of SARS-CoV-2. Am J Respir Crit Care Med. 2020;202(5):756-9. 